\title{
Ultracompact Binaries as Bright X-Ray Sources in Elliptical Galaxies
}

\author{
Lars Bildsten $^{1,2}$ and Christopher J. Deloye ${ }^{2}$
}

\begin{abstract}
Chandra observations of distant $(D \sim 10 \mathrm{Mpc})$ elliptical galaxies have revealed large numbers of Low Mass X-ray Binaries (LMXBs) accreting at $\dot{M}>10^{-8} M_{\odot} \mathrm{yr}^{-1}$. The majority of these LMXBs reside in globular clusters (GCs) and it has been suggested that many of the field LMXBs also originated in GCs. We show here that ultracompact binaries with orbital periods of 8-10 minutes and $\mathrm{He}$ or $\mathrm{C} / \mathrm{O}$ donors of $0.06-0.08 M_{\odot}$ naturally provide the observed $\dot{M}$ 's from gravitational radiation losses alone. Such systems are predicted to be formed in the dense GC environment, a hypothesis supported by the 11.4 minute binary $4 \mathrm{U}$ 1820-30, the brightest persistent LMXB in a Galactic GC. These binaries have short enough lifetimes $\left(<3 \times 10^{6}\right.$ years $)$ while bright $\left(L>10^{38} \mathrm{erg} \mathrm{s}^{-1}\right)$ that we calculate their luminosity function under a steadystate approximation. This yields a luminosity function slope in agreement with that observed for $6 \times 10^{37} \mathrm{erg} \mathrm{s}^{-1}<L<5 \times 10^{38} \mathrm{erg} \mathrm{s}^{-1}$, encouraging us to use the observed numbers of LMXBs per GC mass to calculate the accumulated number of ultracompact binaries. For a constant birthrate over 8 Gyrs, the number of ultracompact binaries which have evolved through this bright phase is $\sim 4000$ in a $10^{7} M_{\odot} \mathrm{GC}$, consistent with dynamical interaction calculations. Perhaps most importantly, if all ultracompacts become millisecond radio pulsars, then the observed normalization agrees with the inferred number of millisecond radio pulsars in 47 Tuc and Galactic GCs in general.
\end{abstract}

Subject headings: accretion, accretion disks - globular clusters: general - gravitational waves - stars: neutron - X-rays: binaries, galaxies

\footnotetext{
${ }^{1}$ Kavli Institute for Theoretical Physics, Kohn Hall, University of California, Santa Barbara, CA 93106; bildsten@kitp.ucsb.edu

${ }^{2}$ Department of Physics, Broida Hall, University of California, Santa Barbara, CA 93106; cjdeloye@physics.ucsb.edu
} 


\section{Introduction}

The Einstein observatory allowed for the discovery and study of X-ray emission from elliptical and S0 galaxies (e.g. Forman, Jones \& Tucker 1985), finding that the emission likely had two distinct contributors (Trinchieri \& Fabbiano 1985, Canizares, Fabbiano, \& Trinchieri 1987). The softer emission is from hot interstellar gas (Forman et al 1985, Trinchieri, Fabbiano, \& Canizares 1986) and dominates the total X-ray emission from the most massive ellipticals. Observations with ASCA (Matsumoto et al. 1997) showed that a harder (5-10 $\mathrm{keV}$ ) spectral component scaled linearly with the bolometric luminosity of the galaxy, confirming its suggested origin as the unresolved contributions of numerous Low Mass X-Ray Binaries (LMXBs; Trinchieri \& Fabbiano 1985; White, Nagase, \& Parmar 1995; Irwin \& Sarazin 1998).

The exquisite point source sensitivity of Chandra led to the discoveries of the expected LMXBs (e.g. Sarazin, Irwin \& Bregman 2000; Blanton, Sarazin \& Irwin 2000; Sarazin, Irwin, \& Bregman 2001; Finoguenov \& Jones 2001; Angelini, Loewenstein, \& Mushotzky 2001; Kraft et al. 2001; Kundu, Maccarone, \& Zepf 2002; Di Stefano et al. 2003; Randall, Sarazin \& Irwin 2004). These observations (see Gilfanov 2004 and Kim \& Fabbiano 2004) typically find 20-50 LMXBs with $L>10^{38} \mathrm{erg} \mathrm{s}^{-1}$ from elliptical galaxies with stellar masses of $(1-3) \times 10^{11} M_{\odot}$. This has provided a large sample of very bright LMXBs that is unattainable from the Milky Way or even M31, allowing for a new probe of binary evolution. Gilfanov (2004) showed that the LMXBs of these elliptical and S0 galaxies are consistent with a single luminosity function

$$
\frac{d N}{d L} \propto \frac{1}{L^{\alpha}}
$$

from $10^{37} \mathrm{erg} \mathrm{s}^{-1}$ to a break value $L_{B}=5_{-0.7}^{+1.4} \times 10^{38} \mathrm{erg} \mathrm{s}^{-1}$. with $\alpha=1.64 \pm 0.22$. Kim and Fabbiano (2004) recently found a similar result for $L>6 \times 10^{37} \mathrm{erg} \mathrm{s}^{-1}$ of $\alpha=1.80 \pm 0.2$ and $L_{B}=4.8 \pm 1.2 \times 10^{38} \mathrm{erg} \mathrm{s}^{-1}$. For $L>L_{B}$, the luminosity function steepens to $\alpha=2.7 \pm 0.5$. The total number of X-ray sources scales with the galactic mass, with $\approx 20$ sources of $L>10^{38} \mathrm{erg} \mathrm{s}^{-1}$ per $10^{11} M_{\odot}$ (Gilfanov 2004), or a total X-ray luminosity that scales linearly with the $K$ band luminosity as $L(\mathrm{LMXB})=1.5 \pm 0.6 \times 10^{40} \mathrm{erg} \mathrm{s}^{-1}$ for $L_{K}=10^{11} L_{K, \odot}(\mathrm{Kim}$ \& Fabbiano 2004).

Starting with the original observation of NGC 4697 (Sarazin et al. 2001), it has become clear that many (20-70\%) of these LMXBs are residing in globular clusters (Angelini et al. 2001; Kundu et al. 2002; Maccarone, Kundu \& Zepf 2003; Minnitti et al. 2004). Kundu et al. (2002) showed that there was no difference in $d N / d L$ for LMXBs in and out of GCs (both had $\alpha=1.55 \pm 0.15$ and $L_{B} \approx 3 \times 10^{38} \mathrm{erg} \mathrm{s}^{-1}$ ) in NGC 4472 , supporting the suggestion of White, Sarazin and Kulkarni (2002) that most of the bright LMXBs in ellipticals are made 
in GCs. Kundu et al. (2002) showed that nearly 4\% of NGC 4472 GCs host a bright LMXB, with metal rich clusters about 3 times more likely to host an LMXB (confirmed in NGC 4365 and NGC 3115 by Kundu et. al. 2003). Sarazin et al. (2003) studied four galaxies and found that the specific incidence of LMXBs in GCs is about one source with $L>10^{38} \mathrm{erg} \mathrm{s}^{-1}$ per $10^{7} L_{\odot, I}$, so that a star in a GC is about 1000 times more likely to be a donor than a star in the field. Such a large enhancement of the incidence of LMXBs in GCs was first found in the Milky Way (Katz 1975; Clark 1975) and is indicative of the important role of interactions in creating mass transferring binaries in GCs (see Hut et al. 1992 for an overview).

Though numerous LMXBs have been found, the nature of the donor star in this old stellar population remains a mystery. Piro \& Bildsten (2002) showed that for field LMXBs, the simplest way to reach $10^{38} \mathrm{erg} \mathrm{s}^{-1}$ (or $\dot{M}=L R / G M \approx 10^{-8} M_{\odot} \mathrm{yr}^{-1}$ for accretion onto a neutron star of $M=1.4 M_{\odot}$ and $R=10 \mathrm{~km}$ ) is to have a red giant branch star fill the Roche lobe with orbital periods of days or longer. They noted that these wide binaries are nearly always transient accretors, and that multiple Chandra observations would easily identify them (e.g. Kraft et al. 2001). However, calculating the resulting $d N / d L$ is impossible given our poor state of knowledge of the transient duty cycle.

We argue here that the most likely type of mass transferring binary responsible for the bright end of the luminosity function in distant elliptical galaxies (especially those in GCs) are "ultracompact" systems consisting of either a $\mathrm{He}$ or $\mathrm{C} / \mathrm{O}$ white dwarf donor of mass $M_{c} \approx 0.04-0.08 M_{\odot}$ which is filling its Roche lobe in a 5-10 minute orbit with a neutron star. In $\S 2$, we summarize the observational clues that led us to this conjecture and derive the resulting $d N / d L$, showing that the observed power law is naturally explained. In $\S 3$, we use the observed normalizations from Chandra to compare to the predicted formation rate of ultracompacts in GCs from dynamical interactions. We also predict the expected number of millisecond radio pulsars (MSPs) assuming that all ultracompacts become MSPs. We close in $\S 4$ by noting more observational tests, and discussing future work.

\section{Ultracompact Binary Formation and Evolution}

There are a number of facts that support our conjecture. The first is that the brightest LMXB in a galactic GC (see Table 1 in Deutsch, Margon \& Anderson 2000) and the only one with a luminosity comparable to that detected in a distant elliptical is the $L \approx(4-7) \times$ $10^{37} \mathrm{erg} \mathrm{s}^{-1}$ source $4 \mathrm{U} 1820-30$ in NGC 6624. Stella, Priedhorsky \& White (1987) found a coherent 11.4 minute periodicity in $4 \mathrm{U} 1820$-30 with a peak to peak variation in the X-rays of $\approx 3 \%$, which is now securely identified as the orbital period (van der Klis et al. 1993). The observed properties of the thermonuclear flashes from this system are best explained by 
accretion of nearly pure helium (e.g. Bildsten 1995; Strohmayer \& Brown 2002; Cumming 2003). The second clue is that the observed luminosity break, $L_{B}$, is much closer to the Eddington limit of a neutron star (NS) accreting material with two baryons per electron (e.g. He or $\mathrm{C} / \mathrm{O}$ )

$$
L_{\mathrm{Edd}}=\frac{8 \pi G M m_{p} c}{\sigma_{\mathrm{Th}}}=3.5 \times 10^{38} \mathrm{erg} \mathrm{s}^{-1}\left(\frac{M}{1.4 M_{\odot}}\right),
$$

(where $\sigma_{\mathrm{Th}}$ is the Thomson scattering cross section) than accretion of cosmic composition This has been noted previously (e.g. Kim and Fabbiano 2004) but none made the connection to ultracompacts.

The last fact is that ultracompact progenitors (i.e. a NS and white dwarf in a few hour orbit) are vigorously produced in GCs (Verbunt 1987; Davies 1995; Rasio, Pfahl and Rappaport 2000). Verbunt (1987) noted that one way to form these systems is a direct stellar collision of a NS with a red giant that would trigger a common envelope (CE) event (though simulations of Rasio and Shapiro 1991 found no common envelope). The CE would allow for inspiral of the NS and red giant He core (of mass $M_{c, i} \approx 0.1-0.4 M_{\odot}$ ) to short enough orbital periods that gravitational wave losses drive it into contact within a Hubble time and initiate mass transfer. Rasio et al. (2000) showed that a much more likely formation channel is an exchange interaction of the NS with a primordial binary (e.g. Hut, Murphy \& Verbunt 1991; Sigurdsson \& Phinney 1993) when the GC was young. The higher main sequence turnoff mass would then trigger unstable mass transfer and a CE event and inspiral of the He core when the star leaves the main sequence. Some systems formed this way could also be ejected from the GC and appear as LMXBS at a later date when the gravitational radiation losses have driven them into contact.

At these very short orbital periods (1-10 minutes), $\dot{M}$ is set by the rate of angular momentum loss from gravitational radiation

$$
\frac{\dot{J}}{J}=-\frac{32 G^{3} M M_{c}\left(M+M_{c}\right)}{5 c^{5} a^{4}}
$$

where $a$ is the orbital separation. Under the constraint of conservative mass transfer and Roche lobe filling, $R_{c}=0.46 a\left(M_{c} /\left(M+M_{c}\right)\right)^{1 / 3}, \dot{M}$ relates to the angular momentum loss rate as

$$
-\frac{\dot{J}}{J}=-\frac{\dot{M}_{c}}{M_{c}}\left(\frac{5}{6}+\frac{n}{2}-\frac{M_{c}}{M}\right)
$$

where $n=d \log R_{c} / d \log M_{c}$ is the rate of change of the WD radius under mass loss. Bildsten (2002) and Deloye and Bildsten (2003; hereafter DB) calculated the impact of finite entropy on the value of $n$, which is most pronounced at longer orbital periods where the donor is 
even less massive, $\approx 0.01 M_{\odot}$. However, the composition of the WD does matter, as Coulomb physics makes the $\mathrm{C}$ or $\mathrm{O}$ donors more compact than He donors at a given mass and modifies $n$, allowing it to range from $n=-0.1$ to $n=-0.3$ (see Figure 1 ).

Figure 1 shows the relations between $M_{c}, n$ and orbital period as a function of the accretion luminosity, $L$, found by integrating equations (3) and (4) with the WD models of DB and an initial NS mass of $M=1.4 M_{\odot}$. This shows that the companion masses required $\left(M_{c}=0.04-0.08 M_{\odot}\right)$ to reach $3 \times 10^{-8} M_{\odot} \mathrm{yr}^{-1}$ are consistent with the evolution of a system with an initial WD donor mass of $>0.1 M_{\odot}$. We plot the evolution here during the $L<L_{\text {Edd }}$ stage and attribute the observed cutoff in $d N / d L$ to either the impact of superEddington accretion or (as noted below) a predominance of ultracompact progenitors with $0.10-0.12 M_{\odot}$ He cores. We see no reason to attribute the $L>L_{\text {Edd }}$ sources to accretion onto black holes and think it most likely that these sources are ultracompacts accreting via a disk at a super-Eddington rate. The best confirmation of our conjecture would be the discovery of the orbital periodicity in the range of Figure 1.

Ultracompacts evolve through the observable $L$ range in $<10^{7}$ years, a timescale much shorter than either the age of the GCs or any reasonable expectations of a timescale over which the birthrate of mass-transferring systems might change. Hence, their total number is indicative of their current birthrate and we can demand continuity within the accreting population to directly calculate $d N / d L$. We start by combining the evolution equations to yield a simple analytic form for $\dot{M}_{c} \propto-M_{c}^{\beta}$ where $\beta=10 / 3-4 n$ in the limit of $M_{c} \ll M$. For $n=-0.2$, this gives $\dot{M}_{c} \propto-M_{c}^{4.13}$. Integrating this equation (and setting $n$ and $M$ constant) yields $M_{c}$ as a function of time, $t$, since the onset of mass transfer, $t \propto M_{c}^{1-\beta}(1-$ $\left.\left(M_{c, i} / M_{c}\right)^{1-\beta}\right)$, where $M_{c, i}$ is the initial WD donor mass set by the progenitor scenario, which can range from $\approx 0.1-0.4 M_{\odot}$ for He cores to $\approx 0.6 M_{\odot}$ for $\mathrm{C} / \mathrm{O}$ cores.

In the limit that $M_{c} \ll M_{c, i}$, we get the simple relation $\dot{M} \propto(1 / t)^{\beta /(\beta-1)}$. The amount of time the binary spends with a luminosity in excess of $L$ is $t \propto(1 / L)^{(\beta-1) / \beta}$, which immediately yields $N(>L) \propto t$. The resulting value of $\alpha$ (in equation 1 ) is then

$$
\alpha=\frac{\beta-1}{\beta}+1=\frac{17-24 n}{10-12 n},
$$

or $\alpha=1.77$ for $n=-0.25$, in excellent agreement with the observed values of $\alpha=1.64 \pm 0.22$ (Gilfanov 2004) and $\alpha=1.8 \pm 0.2$ (Kim and Fabbiano 2004). Clearly, there is little difference in $\alpha$ for the range of $n$ expected from varied compositions. The power law steepens for $M_{c}$ close to $M_{c, i}$, which could explain the observed break at $L_{B}$ if nearly all of the ultracompact progenitors had $M_{c, i} \approx 0.10-0.12 M_{\odot}$. This mass is the He core mass at the turnoff, but we know of no dynamical calculations that point to such a narrow $M_{c, i}$ distribution.

Most importantly, we have shown that the luminosity function slope is nearly indepen- 
dent of both the age of the GC and the type of initial WD donor (i.e. He or C/O). This universality and remarkable agreement with the observations allows us to use the Chandra derived incidence of LMXBs to calculate the ultracompact birthrates and the total number of such systems made over a Hubble time.

\section{Binary Interactions and Millisecond Radio Pulsars in Globular Clusters}

The identification of elliptical LMXBs with ultracompact binaries has broad repercussions. The simplest one to state is that the much smaller donor mass (compared to main sequence donors) naturally shortens the LMXB lifetimes and dramatically increases the implied LMXB birthrate. In their study of four elliptical galaxies, Sarazin et al (2003) found a specific LMXB incidence within GCs of $\approx 1$ source with $L>10^{38} \mathrm{erg} \mathrm{s}^{-1}$ per $10^{7} L_{\odot, I}$, consistent with the $\approx 10^{-7} / M_{\odot}$ found by Kundu et al. (2003). This incidence is independent of GC mass, but dependent on GC metallicity (Kundu et al. 2003). An ultracompact at $L=10^{38} \mathrm{erg} \mathrm{s}^{-1}$ has $\dot{M} \approx 8.4 \times 10^{-9} M_{\odot} \mathrm{yr}^{-1}$, a He donor of $M_{c} \approx 0.06 M_{\odot}$ and an age of $2 \times 10^{6}$ years, implying an ultracompact birthrate of one new bright LMXB every $2 \times 10^{6}$ years in a $10^{7} M_{\odot} G C$.

Kundu et al.'s (2003) preliminary study found that the incidence of bright LMXBs is consistent with being independent of GC age over the range of 3-11 Gyr, allowing us to integrate the birthrate over 8 Gyr to obtain $\sim 4000$ WD-NS binaries capable of undergoing stable mass transfer in a $10^{7} M_{\odot}$ cluster. Davies' (1995) calculations found 5-50 WD-NS binaries that will make contact within 10 Gyrs per 1000 initial binaries. If the initial binary fraction was 10\%, then the observed rate is consistent with Davies (1995) lower estimate. Ivanova and Rasio (2004) found $\sim 30$ WD-NS capable of making contact (with a roughly constant rate in time, see Rappaport et. al. 2001 for a declining rate in time) during the evolution of a cluster model with a core density of $10^{5} M_{\odot} \mathrm{pc}^{-3}$ (similar to 47 Tuc) and a total mass after a Hubble time of $2 \times 10^{5} M_{\odot}$; a factor of three less than implied by the Chandra observations and our identification with ultracompacts. Future comparisons must take into account the initial WD mass and its impact on the binary's fate once contact is established (i.e. merging versus stable mass transfer).

We can also check our hypothesis by comparing these total numbers to the supposed endpoints of the mass transfer, a recycled MSP either in a tight binary (Rasio et al. 2000) or isolated (see Bildsten's (2002) discussion of the likelihood of this). Kulkarni, Narayan and Romani (1990) calculated that the MSP birthrate in all galactic GCs $\left(\sim 10^{8} M_{\odot}\right)$ was $\sim 1$ every $10^{6}$ years for a total number of GC MSPs of $\sim 10^{4}$. Assuming that the X-ray lifetimes of the GC LMXBS was $10^{9}$ years (consistent with mass transfer on the main sequence, but 
likely inconsistent with the lifetime implied by the calculated formation rate; Hut, Murphy \& Verbunt 1991) and using the $\sim 10$ persistent sources, they found a GC LMXB birthrate of one every $10^{8}$ years. They noted that a factor of 100 reduction in the X-ray lifetime was needed to alleviate the discrepancy with the MSP birthrate. In retrospect, it is clear that the GC LMXB birthrate is completely dominated by the highest $\dot{M}$ source, the ultracompact $4 \mathrm{U} 1820-30$ with an age of $2.5 \times 10^{6}$ years. Though the numbers are small, its mere existence gives a GC LMXB birthrate a factor of 100 higher. Rather than depend on 4U 1820-30 to derive the rate, if we use the rate found in elliptical GCs, then there should be $\approx 40,000$ MSPs in all of the galactic GC's, consistent with Kulkarni et al.'s (1990) estimate.

Finally, we compare the expectations from the ultracompact formation rate with the MSP population in the galactic GC 47 Tuc. The discovery of 20 MSPs in 47 Tuc (Camilo et al. 2000) led Camilo et al. (2000) to speculate that there might be as many as 200 MSPs in this $10^{6} M_{\odot}$ cluster. Later X-ray observations (Grindlay et al. 2002; Edmonds et al. 2003) bring this number down to 100 (Camilo, F., priv. comm.). The implied rate from ellipticals would yield $\approx 400$ MSPs, just slightly high.

\section{Summary and Future Work}

Our identification of these distant LMXBs with ultracompact binaries naturally explains the observed luminosity function from Chandra observations and yields the LMXB birthrate of one new mass transferring binary every $2 \times 10^{6}$ years per $10^{7} M_{\odot}$ of GCs. Rather remarkably, this derived birthrate from distant ellipticals agrees with both dynamical calculations (e.g. Ivanova \& Rasio 2004) and the observed number of MSPs in galactic GCs, especially 47 Tuc. The much lower donor mass $\left(\approx 0.06 M_{\odot}\right)$ has alleviated the "birthrate" problem often discussed for LMXBs and MSPs in galactic GCs (e.g. Kulkarni et al. 1990).

The simplest way to prove this hypothesis is to find the 5-10 minute orbital periods. If we use $4 \mathrm{U} 1820-30$ as our example, the level of orbital variability in the X-rays could be as low as a few percent, allowing for Chandra searches amongst the few bright LMXBs in M31 GCs while XMM-Newton could probe much deeper. The source 4U 1820-30 cycles in luminosity by about a factor of 3 over a 171 day cycle for unknown reasons (Chou \& Grindlay 2001). Such variability is easily detected by Chandra, and indeed variability at this level has been reported for bright LMXBs in M31 (e.g. Trudolyubov \& Priedhorsky 2004). However, attributing such behavior as unique to ultracompacts is much harder. Ultraviolet observations of 4U 1820-30 (King et al 1993; Arons and King 1993) confirmed the 11.4 minute orbit and similar work could be done with HST amongst the bright systems in M31. 
The lower luminosities that are visible in the GC sources in our galaxy and in M31 have yet to be probed by Chandra in distant ellipticals. For ultracompact binaries, the expectation is that our derived $d N / d L$ will continue until a $L$ is reached where the systems become transients (presuming no dramatic episode associated with MSP turn-on). DB showed that in the absence of X-ray heating in the outer disk, this would occur at $L \sim 10^{37} \mathrm{erg} \mathrm{s}^{-1}$, whereas with X-ray heating, the disks can remain stable down to much lower X-ray luminosities. Unfortunately, the current populations of ultracompacts in our galaxy don't provide a stringent test of which case is correct. Chandra may be able to identify this cutoff with deeper observations of nearby ellipticals.

There is still much to explain. Clearly, not all bright LMXBs are ultracompact binaries, both because the MSPs in wide binaries with He WDs (orbital periods longer than a day) cannot be made from ultracompacts and because roughly half the galactic GC sources are clearly hydrogen accretors (Kuulkers et al. 2003). However, the strong expectation is that most of the $\mathrm{H}$ donors with $L$ large enough to detect at $10 \mathrm{Mpc}$ are transient accretors (e.g. Piro \& Bildsten 2002), making secure predictions of $d N / d L$ difficult. Observations of the bright LMXBs in M31 (Di Stefano et al. 2002; Trudolyubov \& Priedhorsky 2004) have motivated a stable mass transfer scenario involving thermal timescale mass transfer from evolved stars (Di Stefano et al. 2002), but $d N / d L$ was not derived. We have also not explained the higher incidence of bright LMXBs in metal-rich GCs (Kundu et al. 2002; Maccarone et al. 2004).

We thank F. Camilo, D. Chakrabarty, D. Fox, V. Kalogera, T. Maccarone, S. Phinney, F. Rasio, R. Rutledge, and S. Zepf for discussions. L.B. thanks the Center for Gravitational Wave Physics at Penn State (supported by the NSF under cooperative agreement PHY0114375) for organizing a workshop that triggered this idea, Caltech for hospitality during the writing of the article, and S. Phinney for many helpful discussions. This work was supported by the NSF under grants PHY99-07949 and AST02-05956.

\section{REFERENCES}

Angelini, L., Loewenstein, M. \& Mushotzky, R. F. 2001, ApJ, 557, L35

Arons, J. \& King, I. R. 1993, ApJ, 413, L121

Bildsten, L. 1995, ApJ, 438, 852

Bildsten, L. 2002, ApJ, 577, L27

Blanton, E. L., Sarazin, C. L. \& Irwin, J., A., 2000, ApJ, 552, 106 
Camilo, F., Lorimer, D. R., Freire, P., Lyne, A. G., \& Manchester, R. N. 2000, ApJ, 535, 975

Canizares, C. R., Fabbiano, G., \& Trinchieri, G. 1987, ApJ, 312, 503

Chou, Y. \& Grindlay, J. E. 2001, ApJ, 563, 934

Clark, G. W. 1975, ApJ, 199, L143

Cumming, A. 2003, ApJ, 595, 1077

Davies, M. B. 1995, MNRAS, 276, 887

Deloye, C. J. \& Bildsten, L. 2003, ApJ, 598, 1217 (DB)

Deutsch, E. W., Margon, B. \& Anderson, S. F. 2000, ApJ, 530, L21

Di Stefano, R., Kong, A. K. H., Garcia, M. R., Barmby, P., Greiner, J., Murray, S. S., \& Primini, F. A. 2002, ApJ, 570, 618

Di Stefano, R., Kong, A. K. H., VanDalfsen, M. L., Harris, W. E., Murray, S. S. \& Delain, K. M. 2003, ApJ, 599, 1067

Edmonds, P. D., Gilliland, R. L., Heinke, C. O., \& Grindlay, J. E. 2003, ApJ, 596, 1197

Finoguenov, A. \& Jones, C. 2001, ApJ, 547, L107

Forman, W., Jones, C. \& Tucker, W. C. 1985, ApJ, 293, 102

Gilfanov, M. 2004, to appear in MNRAS, astro-ph/0309454

Grindlay, J. E., Camilo, F., Heinke, C. O., Edmonds, P. D., Cohn, H. \& Lugger, P. 2002, ApJ, 581, 470

Hut, P. , Murphy, B. W., \& Verbunt, F. 1991, A\&A, 241, 137

Hut, P., McMillan, S.,Goodman, J., Mateo, M., Phinney, E. S., Pryor, C., Richer, H.B., Verbunt, F. \& Weinberg, M. 1992, PASP, 104, 981

Irwin, J.A. \& Sarazin, C. L. 1998, ApJ, 494, L33

Ivanova, N., \& Rasio, F.A. 2004, to appear in Proceedings of IAU Colloquium 194 on "Compact Binaries in The Galaxy and Beyond", eds. G. Tovmassian and E. Sion, astro$\mathrm{ph} / 0402537$

Katz, J. I. 1975, Nature, 253, 698

Kim, D.-W. \& Fabbiano, G. 2004, submitted to ApJ, astroph/0312104

King, I. R. et al. 1993, ApJ, 413, L117

Kraft, R. P., Kregenow, J. M., Forman, W. R., Jones, C. \& Murray, S. S. 2001, ApJ, 560, 675 
Kulkarni, S. R., Narayan, R. \& Romani, R. W. 1990, ApJ, 356, 174

Kundu, A., Maccarone, T. J., \& Zepf, S. E., 2002, ApJ, 574, L5

Kundu, A., Maccarone, T. J., Zepf, S. E., \& Puzia, T. H., 2003, ApJ, 589, L81

Kuulkers, E., den Hartog, P.R., in'T Zand, J. J. M., Verbunt, F. W. M., Harris, H. E., and Cocchi, M. 2003, A\&A, 399, 663

Maccarone, T. J., Kundu, A., \& Zepf, S. E. 2003, ApJ, 586, 814

Maccarone, T. J., Kundu, A., \& Zepf, S. E. 2004, to appear in ApJ, astro-ph/0401333

Matsumoto, H., Koyama, K., Awaki, H., Tsuru, T., Loewenstein, M., \& Matsushita, K. 1997, ApJ, 482, 133

Minnitti, D., Rejkuba, M., Funes, J. G.,\& Akiyama, S. 2004, ApJ, 600, 716

Piro, A. L. \& Bildsten, L. 2002, ApJ, 571, L103

Randall, S. W., Sarazin, C. L., \& Irwin, J. A. 2004, ApJ, 600, 729

Rappaport, S., Pfahl, E., Rasio, F. \& Podsiadlowski, P. 2001, in "Evolution of Binary and Multiple Star Systems", ed. Ph. Podsiadlowski, S. Rappaport, A. R. King, F. D'Antona, and L. Burderi (Astronomical Society of the Pacific: San Francisco), p. 409

Rasio, F. A. \& Shapiro, S. L. 1991, ApJ, 377, 559

Rasio, F. A., Pfahl, E. D. \& Rappaport, S. 2000, ApJ, 532, L47

Sarazin, C. L., Irwin, J. A. \& Bregman, J. N. 2000, ApJ, 554, L101

Sarazin, C. L., Irwin, J. A. \& Bregman, J. N. 2001, ApJ, 556, 533

Sarazin, C. L., Kundu, A., Irwin, J. A., Sivakoff, G. R., Blanton, E. L., \& Randall, S. W. 2003, ApJ, 595, 743

Sigurdsson, S. \& Phinney, E. S. 1993, ApJ, 415, 631

Stella, L., Priedhorsky, W., \& White, N. E. 1987, ApJ, 312, L17

Strohmayer, T. E. \& Brown, E. F. , 2002, ApJ, 566, 1045

Trinchieri, G., Fabbiano, G. \& Canizares, C. R. 1986, ApJ, 310, 637

Trinchieri, G., \& Fabbiano, G. 1985, ApJ, 296, 447

Trudolyubov, S. \& Priedhorsky, W. 2004, astro-ph/0402619

van der Klis, M., Hasinger, G., Verbunt, F., van Paradijs, J., Belloni, T., \& Lewin, W. H. G. 1993, A\&A, 279, L21

Verbunt, F. 1987, ApJ, 312, L23 
White, N. E., Nagase, F., \& Parmar, A. N. 1995, in X-Ray Binaries, ed. W. Lewin, J. van Paradijs, \& E. van den Heuvel (Cambridge: Cambridge Univ. Press), 1

White, R. E., Sarazin, C. L. \& Kulkarni, S. R. 2002, ApJ, 571, L23 


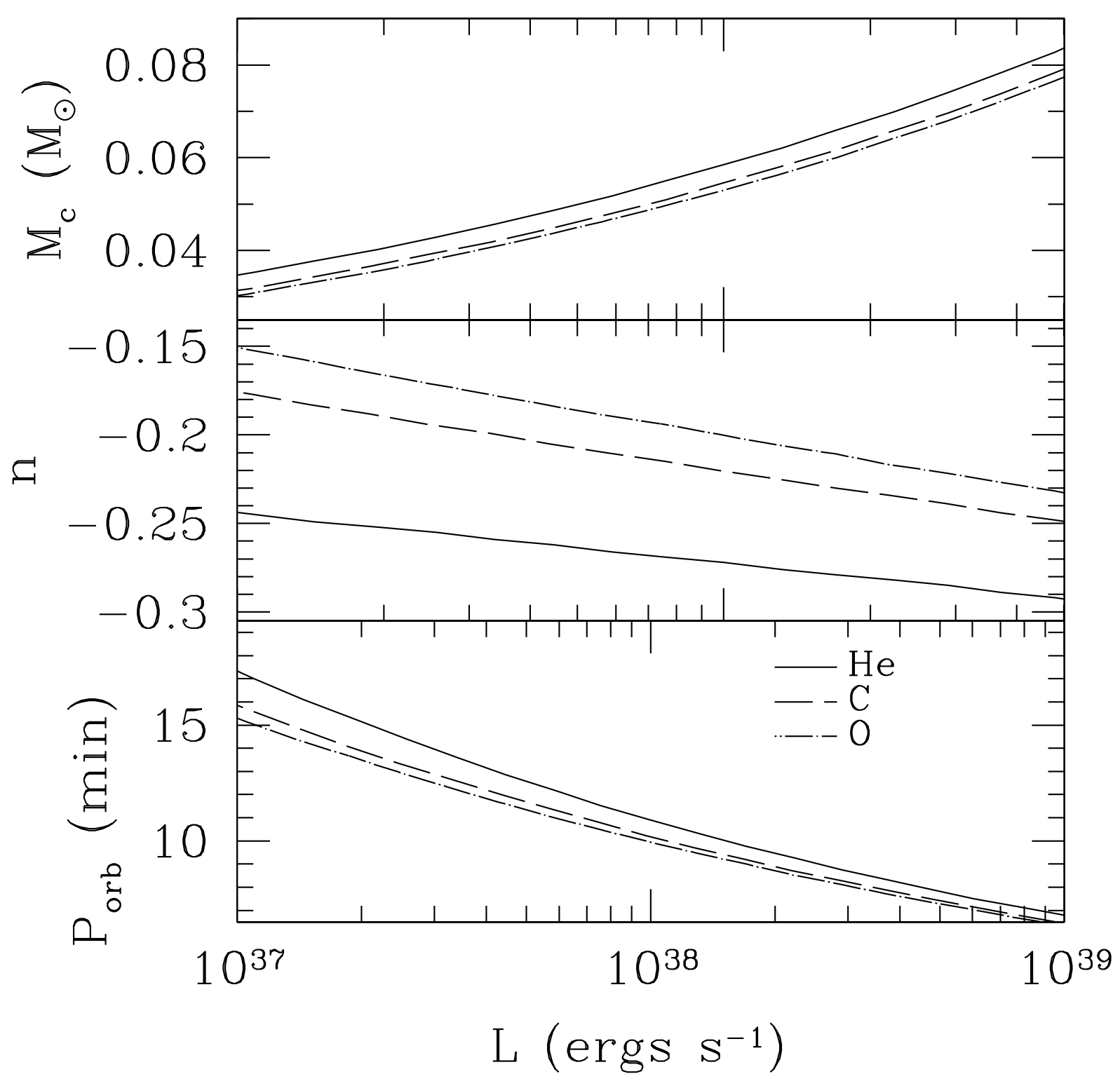

Fig. 1.- The relation between donor mass, $n$, and orbital period as a function of $L$ for cold donors of different compositions (DB) and a NS mass of $1.4 M_{\odot}$. We neglected entropy effects since those modifications are small compared to those from composition differences, which are clearly important. The solid line is for a Helium donor, whereas the dashed is for Carbon and dot-dashed for Oxygen. 